\title{
Spontaneous carotid cavernous fistula in Ehlers Danlos syndrome
}

\author{
R FOX, ${ }^{*}$ F M POPE, $\dagger$ P NARCISI, $\dagger$ A C NICHOLLS, $\dagger$ B E KENDALL $\ddagger$ \\ M D HOURIHAN,§ D A S COMPSTON*
}

From the Section of Neurology* and Department of Radiology, $\S$ University of Wales College of Medicine, Cardiff, the Dermatology Research Group, $†$ Clinical Research Centre, Harrow and the National Hospital for Nervous Diseases, $\ddagger$ London, $U K$

SUMMARY A patient is described with Ehlers Danlos syndrome presenting with spontaneous carotid cavernous fistula, in whom there was biochemical evidence for defective type III collagen synthesis. Despite the risks associated with arterial manipulation, the fistula was successfully closed by interventional neuroradiology and the patient has since remained well. This outcome is in contrast with the results in previous reports.

Type IV Ehlers Danlos syndrome is characterised clinically by skin fragility, joint hypermobility and arterial aneurysms; clinically this disorder is heterogenous and several sub-types are recognised. ${ }^{1}$ These syndromes result from type III collagen deficiency which should be considered even when arterial disease occurs at a single site, since methods of investigation and treatment which are relatively safe in other circumstances may increase vascular damage in individuals with collagen gene mutations. We describe a case of Ehlers Danlos syndrome type IV, presenting with a spontaneous carotid cavernous fistula successfully treated by interventional neuroradiology, in whom type III collagen deficiency was demonstrated.

\section{Case report}

PW (born August 1964) developed spontaneous pulsating tinnitus in his left ear during February 1985; he discovered accidentally that this noise could be stopped by firm pressure over the left side of his neck. Left-sided periorbital swelling, reddening of the conjunctiva and ocular pain developed 6 months later. Examination showed a $3 \mathrm{~mm}$ left-sided pulsatile proptosis with dilated episcleral vessels; there was a loud vascular bruit heard maximally over the left orbit and obliterated by ipsilateral carotid occlusion. He had clinical

Address for reprint requests: Professor D A S Compston, Section of Neurology, University Hospital of Wales, College of Medicine, Heath Park, Cardiff CF4 4XN, UK.

Received 4 August 1987 and in revised form 4 December 1987. Accepted 23 January 1988 features of Ehlers Danlos syndrome type IV including prominent eyes, thin nose and lips, hyperextensible joints, lax thin skin with prominent venous markings, and small linear telangiectases on the trunk. He had previously undergone orthopaedic surgery without complication and there was a history of rectal bleeding associated with multiple colonic diverticulae.

Fibroblast cultures were obtained from explants of a punch skin biopsy, maintained in Dulbecco's medium and labelled with ${ }^{14} \mathrm{C}$ proline. After incubation for 24 hours at $37^{\circ} \mathrm{C}$ in $5 \% \mathrm{CO}_{2}$ the medium and cell layers were separated, acidified and pepsin digested. After precipitation the synthesised collagens were washed, lyophilised and stored prior to gel electrophoresis. Samples were then dissolved, denatured and separated on a $6 \%$ polyacrylamide gel using a tris glycine buffer system. Type III collagen chains were resolved by reduction of disulphide bonded $\alpha$ I(III) ${ }_{3}$ chains after an initial period of electrophoresis allowing $\alpha \mathrm{I}(\mathrm{I})$ and $\alpha 2(\mathrm{I})$ monomers to migrate into the gel. Radioactive proteins were visualised by fluorography and quantitated by scanning densitometry. Separation of radiolabelled collagen monomers (fig 1) showed a clear difference between profiles of the control line and medium or cell layer fractions from the patient. Collagens detected in medium showed a reduction in intensity of the type III band which appeared to run as a variable doublet of slightly higher than normal molecular weight. The cell layer showed abnormal retention of type III collagen. Although symptoms had not occurred in any other family member, PW's mother and only sibling (male) showed similar morphometric and biochemical abnormalities.

Computed tomography showed an enhancing vascular lesion behind the left orbit. A left internal carotid angiogram was performed by right femoral artery catheterisation; the 

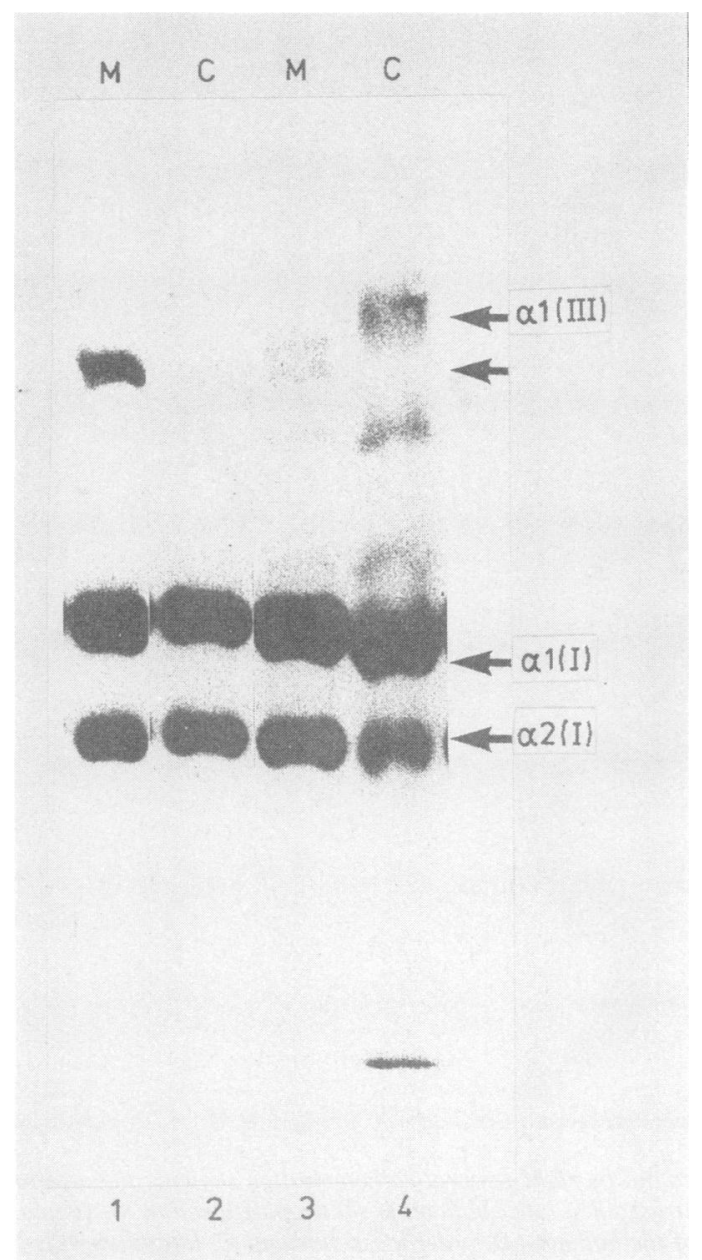

Fig 1 Radioactive tritium labelled pepsinised collagens separated by polyacrylamide gel electrophoresis. Type III collagen runs higher up the gel than $\alpha 1$ and $\alpha 2$ components of type I collagen. Tracks 1 and $2=$ normal control. Tracks 3 and $4=P W . M=$ medium and $C=$ cell layer. There are diminished amounts of type III collagen found in the patient's medium (track 3) whereas a high molecular weight band is retained in the cell layer (track 4).

cervical segment of the internal carotid artery was dilated and irregular in calibre. An aneurysm projecting medially from the proximal intracavernous segment of the artery had ruptured into the cavernous sinus (fig $2 \mathrm{a}$ ); there was early drainage to orbital veins and through the superior and inferior petrosal sinuses to the internal jugular vein (fig 2b). After an interval of 12 months, a transfemoral Debrun-type coaxial catheter system was used to place a $1 \mathrm{ml}$ capacity detachable latex balloon into the cavernous aneurysm. Inflation with contrast medium confirmed that the balloon was satisfactorily placed to occlude the fistula (fig 2c). Permanent distension of the balloon was then established by replacing the contrast medium with a solidifying mixture of silicone, and the balloon was detached using the coaxial catheter. Angiography subsequently confirmed that the fistula was occluded and the carotid normally patent (figs $2 \mathrm{~d}$ $\&$ e). Both the bruit and orbital pain disappeared immediately after the procedure and PW has remained well during the last 16 months.

\section{Discussion}

Most cases of carotid cavernous fistula follow trauma; spontaneous fistulas have been attributed to rupture of pre-existing intracavernous carotid aneurysms or normal dural arteries and to degenerative or inflammatory vascular disease. ${ }^{2}$ There are five previously reported cases of Ehlers Danlos syndrome presenting as spontaneous carotid cavernous fistula. ${ }^{3-6}$ Angiographic investigation by direct puncture or catheterisation was complicated by local haemorrhage or arterial rupture in four; in two patients the carotid artery was friable during subsequent ligation. In one patient the fistula was closed by transvenous balloon embolisation. All five patients died either during investigation or from arterial rupture occurring later at a site remote from the fistula.

Fortunately, complications of investigation or treatment of the carotid cavernous fistula did not occur in our patient. Treatment by interventional radiology was chosen because of the well-documented risks of direct arterial surgery. However arteriography and balloon embolisation may themselves cause fatal arterial damage in the Ehlers Danlos syndrome. This diagnosis should be considered, before radiological or surgical procedures are carried out, in any patient presenting with spontaneous carotid cavernous fistula, in view of the inherent fragility of large arteries in patients with this group of disorders.

Clinical and biochemical classification of collagen defects is complex since biochemically and genetically distinct conditions may resemble each other phenotypically; conversely, some clinically different diseases share similar biochemical defects. Our patient's fibroblasts, and by implication other cells, synthesise reduced amounts of type III collagen which is retained intracellularly, so affecting mechanical properties of collagen-containing tissues such as the media of medium sized arteries. Abnormal synthesis of type III collagen by cultured skin fibroblasts has previously been reported in almost $50 \%$ of individuals with subarachnoid haemorrhage due to ruptured cerebral aneurysm despite the absence of any clinical features of Ehlers Danlos syndrome. ${ }^{78}$ Therefore, although the pathogenesis of arterial rupture is complex, collagen mutations similar to that described in our patient may be important contributory factors in 


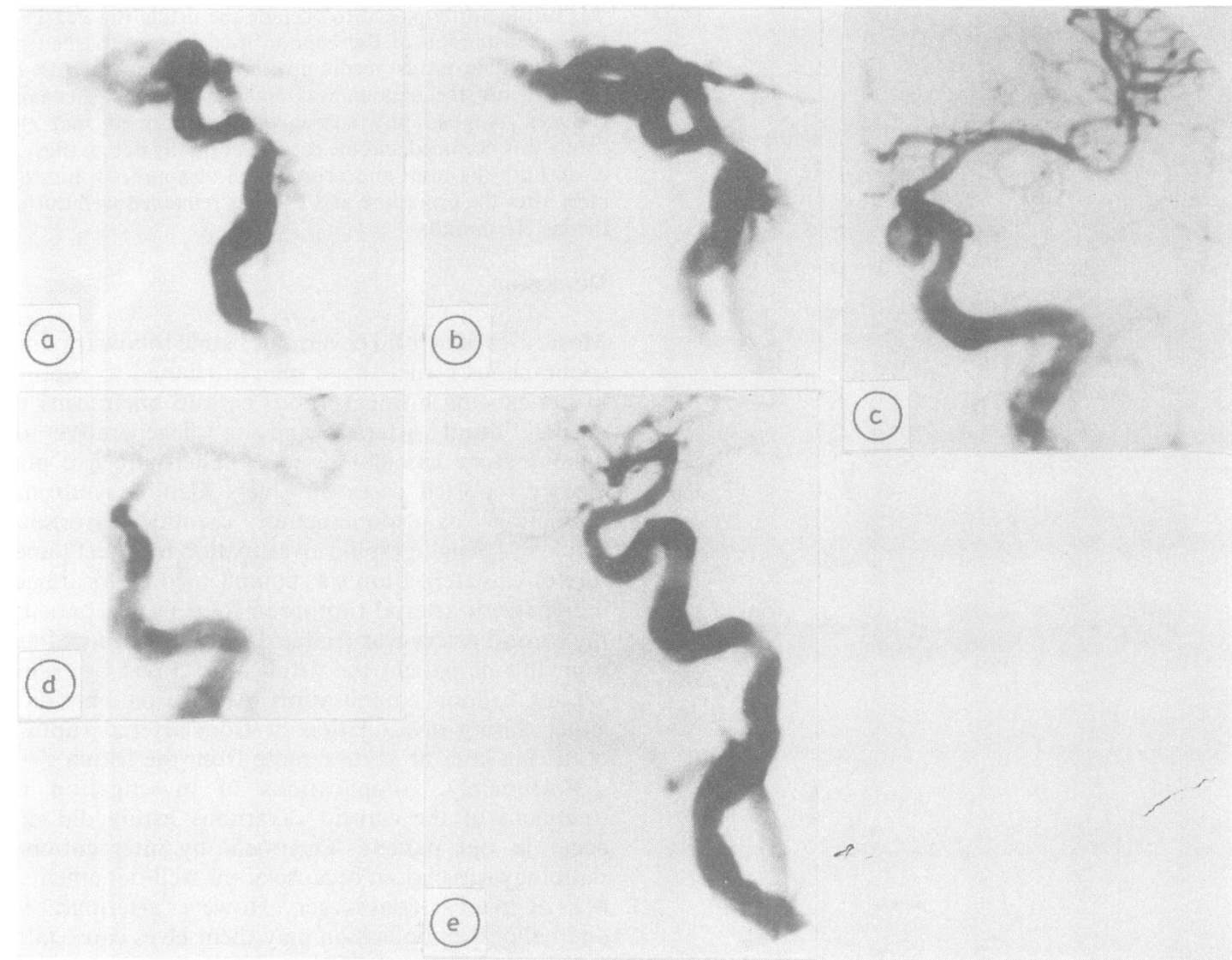

Fig 2 Subtraction views of left internal carotid angiogram carried out by right femoral catheterisation showing the arterial (A: lateral view) and early venous phase (B: lateral view). A contrast filled latex balloon is shown positioned in the fistula (C: anteroposterior view) which is then occluded by detachment of the balloon (D: anteroposterior and E: lateral views).

aneurysm patients lacking specific physical signs of inherited connective tissue disease.

\section{References}

1 Pope FM, Nicholls AC. Collagen genes and proteins in human diseases. In: Champion RH, ed. Recent Advances in Dermatology. London, Churchill Livingstone, 1986:23-52.

2 Barrow DL, Spector RH, Braun IF, Landman JA, Tindall SC, Tindall GT. Classification and treatment of spontaneous carotid-cavernous sinus fistulas. $J$ Neurosurg 1985;62:248-56.

3 Graf CJ. Spontaneous carotid cavernous fistula-Ehlers Danlos syndrome and related conditions. Arch Neurol 1965;13:662-72.
4 Schoolman A, Kepes JJ. Bilateral spontaneous caroticocavernous fistulae in Ehlers Danlos syndrome. J Neurosurg 1967;26:82-5.

5 Julien J, DeBouchard D. Spontaneous caroticocavernous fistula and Ehlers Danlos syndrome. Presse Med 1971;79:1241-2.

6 Farley MK, Clark RD, Fallor MK, Geggel HS, Heckenlively JR. Spontaneous carotico-cavernous fistula and the Ehlers Danlos syndromes. Ophthalmology 1983;90:1337-42.

7 Pope FM, Nicholls AC, Narcisi P, Bartlett J, Neil-Dwyer G, Doshi B. Some patients with cerebral aneurysms are deficient in type III collagen. Lancet 1981;i:973-5.

8 Neil-Dwyer G, Bartlett JR, Nicholls AC, Narcisi P, Pope FM. Collagen deficiency and ruptured cerebral aneurysms; a clinical and biochemical study. J Neurosurg 1983;59:16-20. 